\title{
Modular Modelling of Software Product Lines with Feature Nets ${ }^{\star}$
}

\author{
Radu Muschevici, José Proença, and Dave Clarke \\ DistriNet \& IBBT, Dept. Computer Science, Katholieke Universiteit Leuven, Belgium \\ \{radu.muschevici,jose.proenca, dave.clarke\}@cs.kuleuven. be
}

\begin{abstract}
Formal modelling and verification are critical for managing the inherent complexity of systems with a high degree of variability, such as those designed following the software product line (SPL) paradigm. SPL models tend to be large - the number of products in an SPL can be exponential in the number of features. Modelling these systems poses two main challenges. Firstly, a modular modelling formalism that scales well is required. Secondly, the ability to analyse and verify complex models efficiently is key in order to ensure that all products behave correctly. The choice of a system modelling formalism that is both expressive and well-established is therefore crucial. In this paper we show how SPLs can be modelled in an incremental, modular fashion using a formal method based on Petri nets. We continue our work on Feature Petri Nets, a lightweight extension to Petri nets, by presenting a framework for modularly constructing Feature Petri Nets to model SPLs.
\end{abstract}

\section{Introduction}

The need to tailor software applications to varying requirements, such as specific hardware, markets or customer demands, is growing. If each application variant is maintained individually, the overhead of managing all the variants quickly becomes infeasible 20. Software Produce Line Engineering (SPLE) is seen as a solution to this problem. A Software Product Line (SPL) is a set of software products that share a number of core properties but also differ in certain, well-defined aspects. The products of an SPL are defined and implemented in terms of features, which are subsequently combined to obtain the final software products. The key advantage hereby over traditional approaches is that all products can be developed and maintained together. A challenge for SPLE is to ensure that all products meet their specifications without having to check each product individually, by checking the product line itself. This raises the need for novel SPL-specific formalisms to model SPLs and reason about and verify their properties.

* This research is partly funded by the EU project FP7-231620 HATS: Highly Adaptable and Trustworthy Software using Formal Methods (http://www.hatsproject.eu), and the K.U.Leuven BOF-START project STRT1/09/031 DesignerTypeLab.

G. Barthe, A. Pardo, and G. Schneider (Eds.): SEFM 2011, LNCS 7041, pp. 318 3332011.

(C) Springer-Verlag Berlin Heidelberg 2011 
The main contribution of this paper is a modular modelling framework for specifying the behaviour of software product lines. We use Feature Petri Nets [18, or feature nets (FN) for short, as the modelling formalism. Feature nets are a Petri net extension that enables the specification of the behaviour of an entire software product line (a set of systems) in one single model. The behaviour of an FN is conditional on the features appearing in the product line. The paper makes three contributions. Firstly, it presents a variant of feature nets that improves upon their original definition [18. Secondly, it gives a technique for constructing larger feature nets from smaller ones to model the addition of new features to an SPL. Thirdly, it provides correctness criteria for ensuring that the resulting composition preserves the behaviour of the original model(s).

Organisation: The next section motivates the need for feature nets. Section 3 formally introduces feature nets. Section 4 details an approach at modular modelling with FN, and Section 5 discusses behaviour preservation. Section 6 discusses related work. Section 7 presents our conclusions and future work.

\section{Software Product Line Modelling Challenge}

We illustrate the modelling challenge in software product line engineering (SPLE) using an example of a software product line of coffee vending machines. A manufacturer of coffee machines offers products to match different demands, from the basic black coffee dispenser to more sophisticated machines, such as ones that can add milk or sugar, or charge a payment for each serving. Each machine variant needs to run software adapted to the selected set of hardware features. Such a family of different software products that share functionality is typically developed using an SPLE approach, as one piece of software structured along distinct features. This has major advantages in terms of code reuse, maintenance overhead, and so forth. The challenge is ensuring that the software works appropriately in all product configurations.

Petri nets [17] are used to specify how systems behave. Fig. 1] presents an example of a Petri net for a coffee machine. This has a capacity for $n$ coffee servings; it can brew and dispense coffee, and refill the machine with new coffee supplies. If we now add an optional Milk feature, so that the machine can also

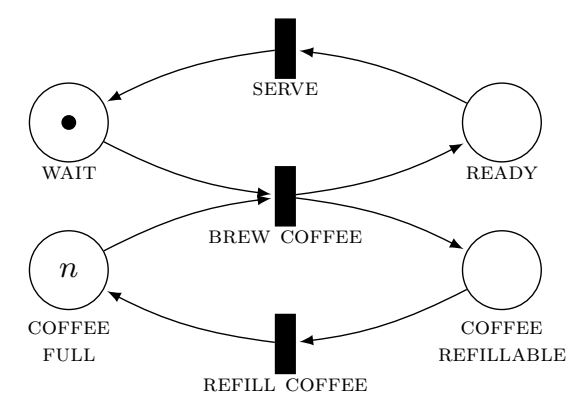

Fig. 1. Petri net model of a basic coffee machine that can only dispense coffee 
add milk to a coffee serving, we need to adapt the Petri net, not only to include the functionality of adding milk, but also to be able to control whether or not this feature is present in the resulting software product.

To address the challenge of modelling a software product line with multiple features, which may or may not be included in any given product, we introduced Feature Petri Nets [18. Feature Petri Net transitions are annotated with application conditions [21, which are propositional formula over features that reflect when the transition is enabled. In this paper we use a variation of Feature Petri Nets in which application conditions are placed on arcs, rather than transitions, called arc-labelled Feature Petri Nets, though we shall just call them feature nets. One advantage of feature nets is that they enable the superposition of the behaviour of the various products (given by different feature selections) in the same model.

Fig. 2 exemplifies a feature net of a coffee machine with a milk reservoir. It considers a product line whose products are over the set of features $\{$ Coffee, Milk $\}$, where Coffee is compulsory and Milk is optional. The application condition above each arc reflects that the arc is present only when the condition evaluates to true. Only then does the arc affect behaviour. If the condition is false, the arc has no effect on behaviour. Consequently, the three transitions on the left-hand side can only fire when the Coffee feature is present; the two transitions on the right-hand side can be taken only when the feature Milk is present. Observe that the restriction of this example net to the transitions that can fire for feature selection $\{$ Coffee $\}$ is exactly the Petri net in Fig. 1, after removing unreachable places.

\section{$3 \quad$ Feature Nets}

A feature net (FN) [18 is a Petri net variant used to model the behaviour of an entire software product line. For this purpose feature nets have application conditions 21] attached to their arcs. An application condition is a propositional logical formula over a set of features, describing the feature combinations to

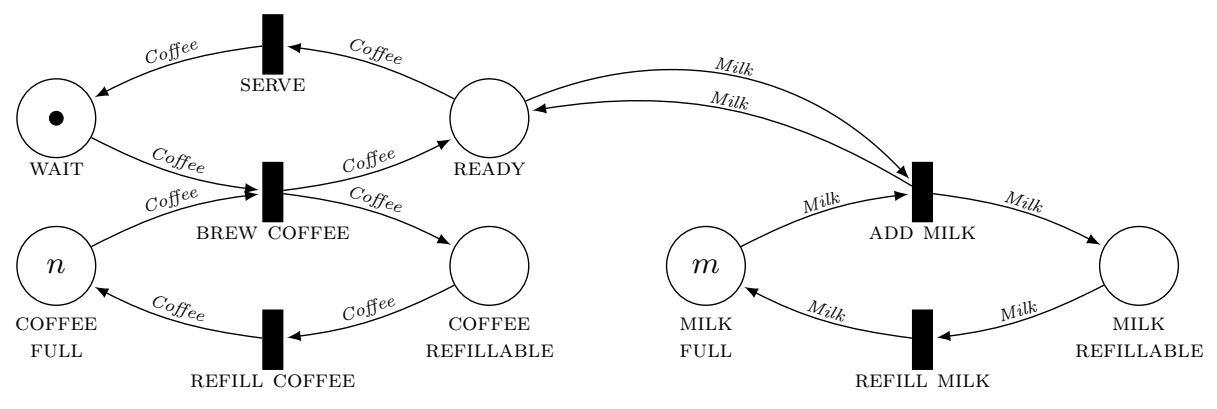

Fig. 2. Feature net of the product line $\{\{C o f f e e\},\{$ Coffee, Milk $\}\}$. Each arc has an application condition attached. 
which the arc applies. If the application condition is false, it is as if the arc were not present.

We define feature nets and give their semantics. We adapt the definition of feature nets described in previous work [18, where application conditions apply to transitions instead of arcs. In that paper two semantic definitions of feature nets were presented. The first semantics directly models the FN for a given feature selection. The second semantics, which we use and adapt here, is given by projecting the $\mathrm{FN}$ for a given feature selection onto a Petri net by removing arcs with unsatisfied application conditions. These two notions have been shown to coincide [18]. We start with some necessary preliminaries, first by defining multisets and basic operations over multisets, then by defining feature nets and their behaviour. Our terminology is standard for Petri nets [8].

Definition 1 (Multiset). A multiset over a set $S$ is a mapping $M: S \rightarrow \mathbb{N}$.

We view a set $S$ as a multiset in the natural way, that is, $S(x)=1$ if $x \in S$, and $S(x)=0$ otherwise. We also lift arithmetic operators to multisets as follows. For any function $\odot: \mathbb{N} \times \mathbb{N} \rightarrow \mathbb{N}$ and multisets $M_{1}, M_{2}$, define $M_{1} \odot M_{2}$ as $\left(M_{1} \odot M_{2}\right)(x)=M_{1}(x) \odot M_{2}(x)$.

Definition 2 (Application condition [21]). An application condition $\varphi$ is a propositional formula over a set of features $F$, defined by the following grammar:

$$
\varphi::=a|\varphi \wedge \varphi| \neg \varphi,
$$

where $a \in F$. Write $\Phi_{F}$ to denote the set of all application conditions over $F$.

Definition 3 (Satisfaction of application conditions). Given an application condition $\varphi \in \Phi_{F}$ and a set of features $F S \subseteq F$, called a feature selection, we say that $F S$ satisfies $\varphi$, written as $F S \models \varphi$, defined as follows:

$$
\begin{array}{lll}
F S \models a & & \text { iff } a \in F S \\
F S \models \varphi_{1} \wedge \varphi_{2} & & \text { iff } F S \models \varphi_{1} \text { and } F S \models \varphi_{2} \\
F S \models \neg \varphi & & \text { iff } F S \not \models \varphi .
\end{array}
$$

Definition 4 (Feature Net). A feature net is a tuple $N=\left(S, T, R, M_{0}, F, f\right)$, where $S$ and $T$ are two disjoint finite sets, $R$ is a relation on $S \cup T$ (the flow relation) such that $R \cap(S \times S)=R \cap(T \times T)=\emptyset$, and $M_{0}$ is a multiset over $S$, called the initial marking. The elements of $S$ are called places and the elements of $T$ are called transitions. Places and transitions are called nodes. The elements of $R$ are called arcs. Finally, $F$ is set of features and $f: R \rightarrow \Phi_{F}$ is a function associating each arc with an application condition from $\Phi_{F}$.

Without $f$ and $F$, a feature net is just a Petri net. Sometimes we omit the initial marking $M_{0}$. The function $f$ determines a node's pre- and post-set, defined below.

Definition 5 (Marking of a feature net). A marking $M$ of a feature net $(S, T, R, F, f)$ is a multiset over $S$. A place $s \in S$ is marked iff $M(s)>0$. 
Definition 6 (Pre-sets and post-sets). Given a node $x$ of a feature net and a feature selection $F S$, the set ${ }^{(F S)} x=\{y \mid(y, x) \in R, F S \models f(y, x)\}$ is the pre-set of $x$ and the $\operatorname{set} x^{(F S)}=\{y \mid(x, y) \in R, F S \models f(x, y)\}$ is the post-set of $x$.

Definition 7 (Enabling). Given a feature selection FS, a marking $M$ enables a transition $t \in T$ if it marks every place in ${ }^{(F S)} t$, that is, if $M \geq{ }^{(F S)} t$.

We now define the behaviour of feature nets for a given feature selection.

Definition 8 (Transition occurrence). Let $N=\left(S, T, R, M_{0}, F, f\right)$ be a feature net and $F S \subseteq F$ a feature selection. A transition $t \in T$ occurs, leading from a state with marking $M_{i}$ to a state with marking $M_{i+1}$, denoted $M_{i} \stackrel{t, F S}{\longrightarrow} M_{i+1}$, iff the following two conditions are met:

$$
\begin{aligned}
& M_{i} \geq{ }^{(F S)} t \\
& M_{i+1}=\left(M_{i}-{ }^{(F S)} t\right)+t^{(F S)} \quad \text { (enabling) }
\end{aligned}
$$

The transition rule for $\mathrm{FN}$ is used to define traces that describe the FN's behaviour. We now define the semantics of a feature net by projecting it onto a Petri net for a given feature selection.

Definition 9 (Projection). Given a feature net $N=\left(S, T, R, M_{0}, F, f\right)$ and a feature selection $F S \subseteq F$, the projection of $N$ onto $F S$, denoted $N \downarrow F S$, is a Petri net $\left(S, T, R^{\prime}, M_{0}\right)$, with $R^{\prime}=\{(x, y) \mid(x, y) \in R, F S \models f(x, y)\}$.

One projects $N$ onto a feature selection $F S$ by evaluating all application conditions $f(x, y)$ with respect to $F S$ for all arcs $(x, y) \in R$. If $F S$ does not satisfy $f(x, y)$, then $(x, y)$ is removed from the Petri net.

The behaviour of a feature net is the union of the behaviour of the Petri nets obtained by projecting all possible feature selections. The behaviour of a Petri net $N=\left(S, T, R, M_{0}\right)$ is given by the set of all of its traces [12, written $\operatorname{Beh}(N)=\left\{M_{0} \stackrel{t_{1}}{\longrightarrow} \cdots \stackrel{t_{s}}{\longrightarrow} M_{n} \mid M_{i} \subseteq S, i \in 1 . . n, M_{i-1} \stackrel{t_{i}}{\longrightarrow} M_{i}\right\}$, and does not include application conditions nor feature selections.

Definition 10 (FN Behaviour). Given an $F N N=\left(S, T, R, M_{0}, F, f\right)$, we define $\operatorname{Beh}(N)$ as follows:

$$
\operatorname{Beh}(N)=\bigcup_{F S \subseteq F} \operatorname{Beh}(N \downarrow F S) .
$$

A feature net combines the behaviour of a set of Petri nets in a single model. Feature nets do not exceed the expressive power of Petri nets. This is indicated by the fact that a feature net can be encoded as a set of Petri nets. Such an encoding involves two steps: first encoding a FN as a transition-labelled Feature Petri Net [18], and secondly describing the behaviour of the Feature Petri Net using a set of regular Petri nets. The first encoding replaces each transition attached to $n$ arcs in $R$ by $2^{n}$ transitions, one for each possible combination of 
the possible arcs. The second encoding step into Petri nets can be achieved by encoding the satisfaction condition of FN transition occurrences by considering for each feature $\mathrm{F}$ two places, $\mathrm{F}$ ON and $\mathrm{F}$ OFF, marked in mutual exclusion depending on whether the feature is selected or not. The details of this encoding are in a previous paper [18.

Given that feature nets are as expressive as Petri nets, analysis techniques for Petri nets still apply to feature nets. At the same time, feature nets offer a concise way to describe the systems in an SPL.

\section{Modular Modelling}

For a modelling formalism to be useful in practice, it needs to facilitate modular development techniques. This is especially important for modelling software product lines: a single SPL model combines the behaviour of a set of different systems, which are often too complex to develop simultaneously.

Modular approaches include top-down techniques, where initially an abstract model is sketched and more details are added incrementally, and bottom-up approaches, where subsystems are modelled separately and later plugged together to a global model. Petri nets support both approaches [12. In the following we propose a bottom-up composition technique for feature nets. It is based on the idea of modelling features of the system individually and then combining them to obtain a model of the entire SPL. Our approach starts by building a model of the core system that is the behaviour which is common to all products of the SPL. Optional features are modelled as separate nets, which also specify how they interact with the core through an interface. Core and additional features are then composed stepwise, by incrementally applying each feature to the core. We show how this technique can be applied to modularly specify a coffee machine product line from the three features Coffee, Payment and Milk.

\subsection{Feature Net Composition}

We devise a modular modelling approach in which features are first expressed as separate FNs. A feature's interaction with the rest of the system (the core) is modelled using an interface. Features are modelled separately in such a way that they can be attached to the core, in order to incrementally build a larger model. The interface simulates the behaviour of the core that the features are designed to be plugged into. A feature modelled using this technique can be seen as a partially specified model of the entire SPL, where the feature's behaviour is fully specified, whereas everything else is underspecified. Composition then entails connecting the interface to the core to obtain a specification of the combined system.

The three features of our example coffee machine are modelled as separate FNs (Fig. 3). Apart from when a feature's behaviour is self-contained (such as the Coffee net in Fig. 3 ) it will typically interact with other features that are part of the larger system. To faithfully model such interactions we include an interface. The aim of the interface is to abstract part of the larger system's 


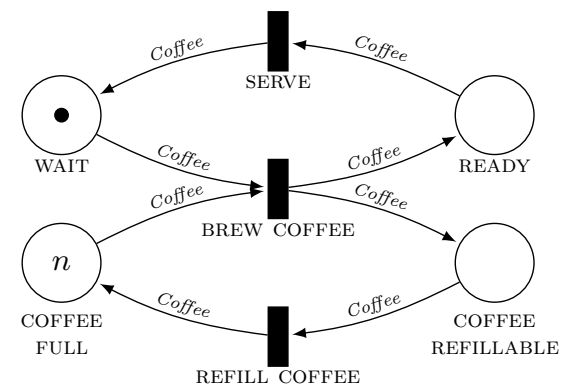

(a) Coffee feature (core)

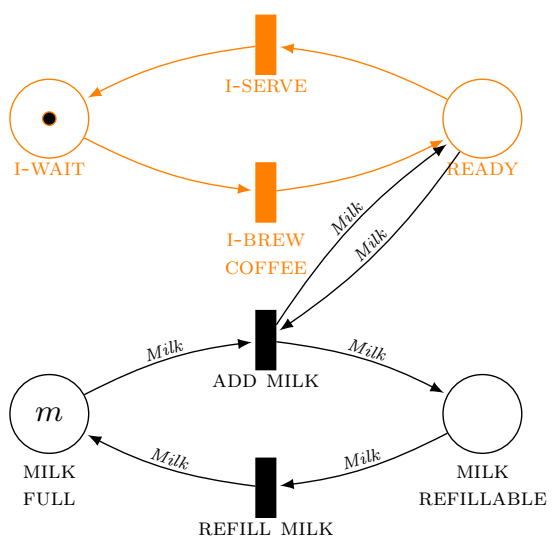

(b) Milk feature

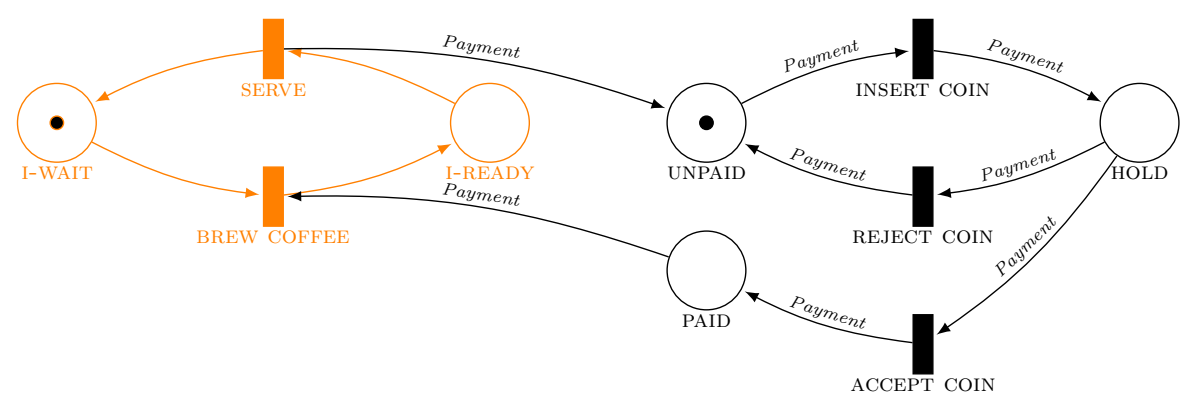

(c) Payment feature

Fig. 3. Individual feature nets modelling the features Coffee, Milk and Payment of a product line of coffee machines. Interfaces are highlighted in orange.

behaviour. The interface will also be used to show that the net exposes the same behaviour as it does when it is part of the combined system. For example, the model of Milk in Fig. $3 \mathrm{~b}$ reflects the fact that adding milk depends on a state of the system in which a cup of fresh coffee is available. The larger system is represented abstractly by the highlighted interface, which models the availability of coffee in the place READY; a token in this place would denote a state in which a freshly brewed cup of coffee is available. Similarly, Fig. 3. models the fact that after a payment has been accepted, the overall system is able to BREW COFFEE, and after serving the coffee, the system goes back to an UNPAID state.

Constructing a model of the whole SPL is done by stepwise applying the delta nets of each feature to a core model. The intuition behind delta net application is that each interface is replaced with a more complex feature net. In our example, the first step could be to refine Payment's interface by replacing it with the feature net for Coffee. In a second step, the feature Milk is refined by replacing its interface with the net obtained in the previous step. 


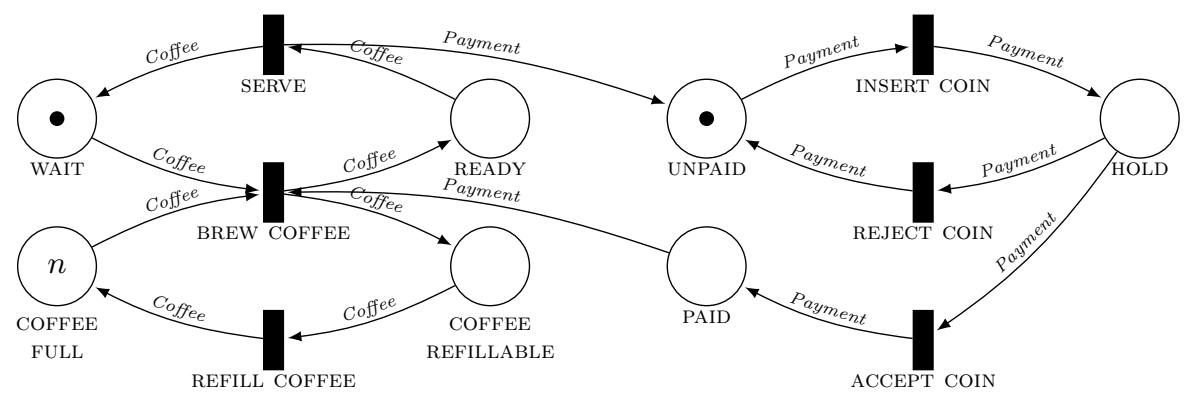

Fig. 4. A software product line over feature set $\{$ Coffee, Payment $\}$ obtained by applying the delta net Payment (Fig. 3r) to the core net modelling Coffee (Fig. 3a)

We now formally introduce the application of a delta net to a core net.

Definition 11 (Delta Feature Net). A delta feature net $N$ is a FN with a designated interface, denoted $N=\left(S, T, R, F, f, S_{I}, T_{I}\right)$, where $S_{I} \subseteq S, T_{I} \subseteq T$.

Delta feature nets specify the behaviour of features designed to be added to a larger system. A set of delta $\mathrm{FN}$ is combined with a stand-alone FN, the core, by sequentially applying each delta net to the core. Delta nets include an interface, which models interactions with the core. Such interactions are modelled by transitions or places common to both core and delta net.

Definition 12 (Delta Net Application). Let $N=(S, T, R, F, f)$ be a feature net and $D=\left(S_{d}, T_{d}, R_{d}, F_{d}, f_{d}, S_{I}, T_{I}\right)$ a delta feature net with $S \cap S_{d} \neq \emptyset$. The application of $D$ to $N$ results in a net $N^{\prime}=\left(S^{\prime}, T^{\prime}, R^{\prime}, F^{\prime}, f^{\prime}\right)$, written as $N \oplus D$,

$$
\text { where } \begin{array}{rlr}
S^{\prime} & =\left(S_{d} \backslash S_{I}\right) \cup S & F^{\prime}=F \cup F_{d} \\
T^{\prime} & =\left(T_{d} \backslash T_{I}\right) \cup T & f^{\prime}=\left(f \cup f_{d}\right) \uparrow R^{\prime} \\
R^{\prime} & =\left\{(s, t) \in\left(R \cup R_{d}\right) \mid s \in S^{\prime}, t \in T^{\prime}\right\} & \\
& \cup\left\{(t, s) \in\left(R \cup R_{d}\right) \mid t \in T^{\prime}, s \in S^{\prime}\right\} . &
\end{array}
$$

When applying a delta net to a core, the interface is dropped and the two nets are "fused" along their common nodes. The arcs that previously connected the delta net interface now connect the core. The applicability of a delta net is limited to certain cores. Let $S_{B}$ and $T_{B}$ represent the border of the interface, that is, $S_{B}=$ $\left\{s \in S_{I} \mid \exists t \in T_{d} \backslash T_{I}:(s, t) \in R^{\prime}\right\}$ and $T_{B}=\left\{t \in T_{I} \mid \exists s \in S_{d} \backslash S_{I}:(t, s) \in R^{\prime}\right\}$. A delta net is applicable to a core net if the border of the interface is preserved, that is, if $S \cap S_{d}=S_{B}$ and $T \cap T_{d}=T_{B}$.

We show how delta net application is used to build a model of the example coffee machines SPL. Starting with the separate sub-models in Fig. 3. delta nets are applied stepwise to a growing core. First, a model with the two features Coffee and Payment is composed by applying the delta net from Fig. 3r to the core shown in Fig. 33. These nets have the two transitions SERVE and BREW COFFEE in common. The result after applying the delta feature net is the new core feature net shown in Fig. 4. In a second step, we add the Milk behaviour by applying the feature net in Fig. 3 b to the core obtained in the previous step. 


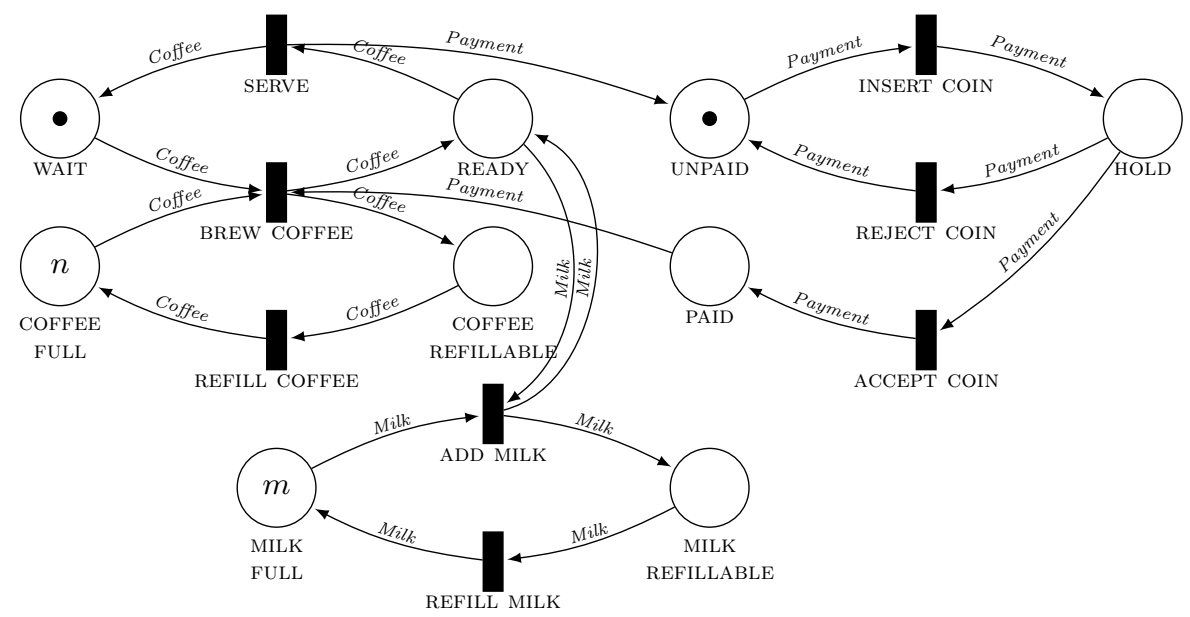

Fig. 5. FN model of an SPL over the feature set $\{$ Coffee, Payment, Milk $\}$ obtained by sequential application of the delta nets for the features Payment (Fig. 3. ) and Milk (Fig. 3b)

These two nets have the place READY in common. The result after delta net application is the model shown in Fig. 5. Note that the order in which we apply the two delta nets does not matter in this case, because neither feature (Milk or Payment) depends on the other. In general, features can depend on other features. This would be reflected by the design of their interfaces, effectively restricting the applicability and ensuring that the delta nets can only be applied in a valid order. As a consequence, delta net application is not commutative.

\section{Correctness}

When is the application of a delta net $D$ to a core net $N$ correct? We consider this application correct if the traces of $N$ and $D$ are in some way the same as the traces of $N \oplus D$, introduced in Definition 12, after projecting onto the transitions of $N$ and $D$. However, there are various ways to compare these traces. We can focus only on the features used by the original nets or on the features used by the combined net. Also checking correctness of the core net can be different from checking correctness of the delta net. Finally, it might be enough to consider only trace inclusion between the original nets and the combined net. The three dimensions are summarised as:

- Original vs. combined features. When comparing the behaviour of one of the original nets with the combined net, we can either consider the combined features in the final net or just the features in one of the original nets.

- Core vs. delta. We can evaluate the correctness of the core or delta net behaviour, always in comparison to the combined net's behaviour.

- Liveness, safety, or both. Liveness states that a net cannot inhibit behaviour in the other net, while safety states that a net cannot introduce new 
behaviour to the other net. For example, we say a delta application is safe with respect to the core net $N$ if the traces of the combined net are included in the traces of $N$, when considering the common transitions.

By choosing different parameters along these dimensions we obtain different notions of correctness. We formulate a parametrised notion of correctness for the application of delta net $D$ to a core net $N$ as follows:

$$
\forall F S \subseteq \Theta_{1}: \operatorname{Beh}\left(\Theta_{2} \downarrow F S\right) \quad \Theta_{3} \quad \operatorname{Beh}((N \oplus D) \downarrow F S) \quad \text { (parametrised correctness) }
$$

where $\Theta_{1}$ can be either the full set of features or the features of the net $\Theta_{2}, \Theta_{2}$ can be either the core or the delta net, and $\Theta_{3}$ is an inclusion or equivalence relation between the two sets of traces, with respect to a set of relevant transitions. When $\Theta_{3}$ is a superset relation, it represents safety, since no new traces can be introduced by combining the two nets. On the other hand, a subset relation represents liveness, since all traces in the original net are still valid traces in the combined net. When we have both safety and liveness assurances, we say that the behaviour is preserved, and instantiate $\Theta_{3}$ to be the equality of the traces with respect to the common transitions.

Not all combinations of these dimensions are desirable in all cases. For example, sometimes we might want to inhibit or extend the behaviour of a core net with respect to the combined set of features, breaking the liveness or safety criteria. However, it seems desirable to preserve this behaviour with respect to the features of the core net. In fact, it is open to debate which combination of these dimensions are ideal. In this paper, we provide sufficient conditions to guarantee:

1. Preservation of the behaviour of $N$ with respect to the original features.

2. Preservation of the behaviour of $D$ with respect to the combined features.

3. Safety of the behaviour of $N$ with respect to the combined features.

\subsection{Mathematical Preliminaries}

We defined liveness and safety as inclusion of traces with respect to a relevant set of traces. We formalise this concept below.

Definition 13 (Behaviour inclusion $\left.\subseteq_{T s}\right)$. Let $N_{i}=\left(S_{i}, T_{i}, R_{i}\right)$ be a pair of Petri nets, for $i \in 1 . .2$, and $T s$ be a set of transitions. We say that the behaviour of $N_{1}$ is included by the behaviour of $N_{2}$ with respect to Ts, written $\operatorname{Beh}\left(N_{1}\right) \subseteq T s \operatorname{Beh}\left(N_{2}\right)$, if $\operatorname{Beh}\left(N_{1}\right)\left\lceil T s \subseteq \operatorname{Beh}\left(N_{2}\right)\lceil T s\right.$, where $\operatorname{Beh}(N)\lceil T s=$ $\{t r\lceil T s \mid \operatorname{tr} \in \operatorname{Beh}(N)\}$ and:

$$
M \uparrow T s=\varepsilon \quad(M \stackrel{t}{\rightarrow} t r) \uparrow T s= \begin{cases}t \cdot(t r \uparrow T s) & \text { if } t \in T s \\ t r \uparrow T s & \text { otherwise. }\end{cases}
$$

Similarly, we write $\supseteq_{T s}$ and $=_{T s}$ to represent superset inclusion and equality for the transitions in $T s$.

We now define weak bisimulation between two feature nets, which we will use to relate the interface of a delta net with the net to which the delta is applied to, based on the notion of bisimulation described by Schnoebelen and Sidorova 22 . 
Definition 14 (Weak bisimulation). Let $N_{i}=\left(S_{i}, T_{i}, R_{i}, M_{0 i}, F_{i}, f_{i}\right)$ be two feature nets, for $i \in 1 . .2, \mathcal{M}_{i}$ the set of markings of $N_{i}$, and $\mathcal{B} \subseteq\left(\mathcal{M}_{1} \times\right.$ $\left.\mathcal{M}_{2}\right) \cup\left(T_{1} \times T_{2}\right)$ a relation over markings and transitions. Recall also the notion of occurrence of transitions introduced in Definition [8, In the following we write $t \in \mathcal{B}$ to denote that $t$ is in the domain or codomain of $\mathcal{B}$. $\mathcal{B}$ is a weak bisimulation if, for any feature selection FS:

1. $M_{01} \mathcal{B} M_{02}$

2. $\forall\left(M_{1}, M_{2}\right) \in \mathcal{B}$, if $M_{1} \stackrel{t_{1}, F S}{\longrightarrow} M_{1}^{\prime}$ and $t_{1} \notin \mathcal{B}$, then $M_{1}^{\prime} \mathcal{B} M_{2}$;

3. $\forall\left(M_{1}, M_{2}\right) \in \mathcal{B}$, if $M_{1} \stackrel{t_{1}, F S}{\longrightarrow} M_{1}^{\prime}$ and $t_{1} \in \mathcal{B}$, then there exists $t_{2} \in T_{2}$ and $M_{2}^{\prime}$ such that $M_{2} \stackrel{t_{2}, F S}{\longrightarrow} M_{2}^{\prime}, M_{1}^{\prime} \mathcal{B} M_{2}^{\prime}$, and $t_{1} \mathcal{B} t_{2}$;

4. conditions (2) and (3) also hold for $\mathcal{B}^{-1}$;

where $M \stackrel{t, F S}{\longrightarrow} \mathcal{B} M^{\prime}$ denotes that there are $n$ transitions $t_{1} \ldots t_{n}$ such that

$$
M \stackrel{t_{1}, F S}{\longrightarrow} \cdots \stackrel{t_{n}, F S}{\longrightarrow} M_{n} \stackrel{t, F S}{\longrightarrow} M^{\prime} \quad \text { and } \quad \forall j \in 1 . . n: t_{j} \notin \mathcal{B} .
$$

If a weak bisimulation exists between $N_{1}$ and $N_{2}$ we say that they are weakly bisimilar, written $N_{1} \approx N_{2}$.

Let $C$ be the feature net for the Coffee feature (Fig. 3k), and $P$ the delta net dealing with Payment (Fig. 35). The interface of $P$ can be seen as a feature net $P_{I}$. It holds that $C \approx P_{I}$. Furthermore, there exists a bisimulation $\mathcal{B}$ that relates the transitions with the same name of the two nets, namely SERVE and BREW COFFEE. More specifically, the relation $\mathcal{B}$ below is a bisimulation, where we write $\mathcal{M}_{C}$ and $\mathcal{M}_{P_{I}}$ to denote all the markings of $C$ and $P_{I}$, respectively.

$$
\begin{aligned}
& \left.\left\{\left(M, M^{\prime}\right) \mid M \in \mathcal{M}_{C}, M^{\prime} \in \mathcal{M}_{P_{i}}, M(\text { WAIT })=1, M^{\prime} \text { (WAIT }\right)=1\right\} \cup \\
& \left.\left\{\left(M, M^{\prime}\right) \mid M \in \mathcal{M}_{C}, M^{\prime} \in \mathcal{M}_{P_{i}}, M(\text { WAIT })=0, M^{\prime} \text { (WAIt }\right)=0\right\} \cup \\
& \{(\text { SERVE, SERVE), (BREW COFFEE, BREW COFFEE) }\}
\end{aligned}
$$

\subsection{Preservation of the Core Behaviour for the Original Features}

Our first criterion compares the core net with the combined net, considering only the features originally present in the core net. We require the behaviour of the core net to be preserved in the combined net, that is, their traces must coincide with respect to the transitions in the core net. We formalise this criterion as follows.

Criterion 1 (preservation/core/original). Let $N=\left(S, T, R, F, M_{0}, f\right)$ be a core net and $D$ a delta net. We say that $N \oplus D$ preserves the behaviour of $N$ for the features in $F$ iff

$$
\forall F S \subseteq F: \operatorname{Beh}(N \downarrow F S)=_{T} \operatorname{Beh}(N \oplus D \downarrow F S) .
$$

To verify that a delta net application obeys the above correctness criteria, it is sufficient (although not necessary) to verify the following condition. Check that 
the arcs between the interface and the non-interface nodes of $D$ require at least one 'new' feature to be present. By new feature we mean a feature that is not in $F$. This syntactic check ensures that, when considering only the features from the core net, the arcs connecting it to the delta net will never be active.

Theorem 1. Let $D=\left(S_{d}, T_{d}, R_{d}, M_{0 d}, F_{d}, f_{d}, S_{I}, T_{I}\right)$ be a delta net, $N=(S, T$, $\left.R, M_{0}, F, f\right)$ a feature net, and $R_{I} \subseteq R_{d}$ be the set of arcs connecting interface nodes $\left(S_{I} \cup T_{I}\right)$ to non-interface nodes. The behaviour of $N$ is preserved by $N \oplus D$ for the features in F (Criterion 11) if:

$$
\forall(x, y) \in R_{I}: \forall F S \in F_{d} \cup F: F S \models f(x, y) \quad \rightarrow \quad F S \cap\left(F_{d} \backslash F\right) \neq \emptyset .
$$

A proof for this theorem can be found in the accompanying technical report 19 . In both our examples of delta applications, that is, adding payment to a coffee machine and adding milk to the resulting net, the condition in Equation (11) holds. The intuition is that, for example, when the Payment feature is not available, the Coffee feature net is detached from the Payment feature net in the combined net. Hence its behaviour is not affected by the Payment net and is preserved.

\subsection{Preservation of the Delta Behaviour for the Combined Features}

We now define the second correctness criterion.

Criterion 2 (preservation/delta/combined). Let $N=\left(S, T, R, M_{0}, F, f\right)$ be a core net and $D=\left(S_{d}, T_{d}, R_{d}, M_{0 d}, F_{d}, f_{d}, S_{I}, T_{I}\right)$ a delta net. We say that $N \oplus D$ preserves the behaviour of $D$ with respect to features from the combined net iff

$$
\forall F S \subseteq F \cup F_{d}: \operatorname{Beh}(D \downarrow F S)==_{T_{d} \backslash T_{I}} \operatorname{Beh}(N \oplus D \downarrow F S) .
$$

As with the correctness Criterion 1, we present a sufficient condition that guarantees the preservation of the Criterion 2. However, as opposed to the previous case, this condition is based on a semantic property of the the interface and the core net.

Theorem 2. Let $D=\left(S_{d}, T_{d}, R_{d}, M_{0 d}, F_{d}, f_{d}, S_{I}, T_{I}\right)$ be a delta net, $N_{I}=$ $\left(S_{I}, T_{I}, R_{I}, M_{0 D}, F_{d}, f_{d}\right)$ be the interface of $D, N=(S, T, R, F, f)$ a (core) feature net, and $R_{B} \subseteq R_{d}$ denote the arcs connecting interface to non-interface nodes. The behaviour of $D$ is preserved by $N \oplus D$ (Criterion 2 ) if $N \approx N_{I}$ and there is a weak bisimulation $\mathcal{B} \subseteq\left(\mathcal{M}_{1} \times \mathcal{M}_{2}\right) \cup\left(T_{1} \times T_{2}\right)$ such that:

$$
\begin{gathered}
\left\{(t, t) \mid t \in T \cap T_{I}\right\} \subseteq \mathcal{B}, \\
\forall s \in S \cap S_{I},\left(M, M^{\prime}\right) \in \mathcal{B}: M(s)=M^{\prime}(s), \\
\forall(s, t) \in R_{B}, s \in S_{I},\left(M, M^{\prime}\right) \in \mathcal{B}:(M-\{s \mapsto 1\}) \mathcal{B}\left(M^{\prime}-\{s \mapsto 1\}\right) \\
\forall(t, s) \in R_{B}, s \in S_{I},\left(M, M^{\prime}\right) \in \mathcal{B}:(M+\{s \mapsto 1\}) \mathcal{B}\left(M^{\prime}+\{s \mapsto 1\}\right)
\end{gathered}
$$

For Equation (44) we assume that, if $M(s)=M^{\prime}(s)=0$, then subtracting $\{s \mapsto 1\}$ does not change the markings. 
The proof for Theorem 2 can be found in the accompanying technical report 19]. Recall our running examples. As explained in the end of Section 5.1, there is a weak bisimulation between the interface of the delta net for payment $P$ and the core net for coffee $C$. This simulation obeys Equation (2) because the shared transitions are related by $\mathcal{B}$, Equation (3) because there places of $C$ and $P$ are disjoint, and Equation (4) because, in this case, $\operatorname{dom}(R) \cap S_{I}=\emptyset$. Hence the composition $C P=C \oplus P$ is correct with respect to Criterion 2. Consider now the application of the delta net for milk $M$ to the previously obtained core $C P$. A possible weak bisimulation between $C P$ and the interface of $M$ relates equal markings of the places READY in $C P$ and READY in $M$, as well as of the places WAIT and I-WAIT. Note that, in order to use Theorem 2 we need to include markings for any number of tokens in READY, because of Equations (44) and (5). Hence, Equation (2) trivially holds, and our specific bisimulation relation also captures Equation (3). We conclude that the composition $C P \oplus M$ is also correct with respect to Criterion 2

\subsection{Safety of the Core Behaviour for the Combined Features}

Our last correctness criterion compares the core net with the combined net with respect to all features, as opposed to the first criterion that only considered the features of the core net. When including the features in the delta net, we consider it safe to inhibit traces that were initially possible, provided that no new traces are introduced. We formalise safety using trace inclusion.

Criterion 3 (safety/core/combined). Let $N=\left(S, T, R, M_{0}, F, f\right)$ be a core net and $D=\left(S_{d}, T_{d}, R_{d}, M_{0 d}, F_{d}, f_{d}, S_{I}, T_{I}\right)$ a delta net. We say that $N \oplus D$ is safe with respect to $D$ and to the combined features iff

$$
\forall F S \subseteq F \cup F_{d}: \operatorname{Beh}(N \downarrow F S) \supseteq_{T} \operatorname{Beh}(N \oplus D \downarrow F S) .
$$

We claim that, when applying a delta net connecting only places from the interface to the rest of the delta, the delta net application is safe with respect to $D$ and the combined features.

Theorem 3. When applying a delta net $D=\left(S_{d}, T_{d}, R_{d}, M_{0 D}, F_{d}, f_{d}, S_{I}, T_{I}\right)$ to a core net $N$, we guarantee that $N \oplus D$ is safe with respect to $D$ and the combined features if:

$$
\forall s \in S_{I}, t \in T_{d} \backslash T_{I}: \quad(s, t) \notin R_{D} .
$$

The theorem is easily justified by the fact that the core net will only be connected to the rest of the delta net through transitions. When the application of a delta net respects Equation (6), we are increasing the pre- and post-sets of these transitions, thereby further restricting when they can be fired.

We exemplify the application of two delta nets in this paper: the Payment and the Milk nets (Fig. 3k and 3b). The first net obeys the condition in Theorem 3 , hence the correctness Criterion 3 holds. The second delta net has arcs connecting places from the interface to a non-interface transition, invalidating Equation (6). However, in this case the safety criterion is preserved, because a token that exits the core when firing ADD MILK is transported back to its origin in the same step. 


\section{Related Work}

Our research relates to Petri net based formalisms, and to the behavioural specification of software product lines. We highlight the most relevant works in these areas. Petri net composition and decomposition strategies that preserve some properties of the initial net(s) have been studied thoroughly [4|23|22|12]. In Open Petri Nets 2], places designated as open represent an interface towards the environment. Open nets are composed by fusing common open places, and the composition operation is shown to preserve behaviour with respect to an inverse decomposition operation. Our Petri net model uses a similar notion of interface, which includes an abstraction of the net that will be matched during application. We use an incremental approach using application of deltas instead of a symmetric composition operation, guided by the intuition that larger systems are build by extending more fundamental systems. The main focus of open Petri nets is the study of properties in a category of nets, while we have a more practical focus on the incremental development of nets. Various formalisms have been adopted for specifying the behaviour of software product lines, with the aim of providing a basis for analysis and verification of such models. A survey of formal methods for software product lines has recently been published [5]. UML activity diagrams have been used to model the behaviour of SPL by superimposing several such diagrams in a single model 7 . Attached to the activity diagram's elements are "presence expressions," which are similar to application conditions. Compared to activity diagrams, Petri nets have a stronger formal foundation, with a larger spectrum of analysis and verification techniques, although, several studies have expressed the semantics of UML diagram using Petri nets (e.g. 10]). Gruler et al. extended Milner's CCS with a product line variant operator that allows an alternative choice between two processes 14 . The PL-CCS calculus includes information about variability: by defining dependencies between features, one can control the set of valid configurations [13]. Variability is often modelled using transition systems enhanced with product-related information. Modal transition systems (MTS) [15] allow optional transitions, lending themselves as a tool for modelling a set of behaviours at once [11. Generalised extended MTS 9] introduce cardinality-based variability operators and propose to use temporal logic formulas to associate related variation points. Asirelli et al. encode MTS using propositional deontic logic formulas and provide a framework for reasoning about behavioural aspects [1]. Modal I/O automata [16] are a behavioural formalism for describing the variability of components based on MTS and I/O automata. Mechanisms for component composition are provided to support a product line theory. These approaches do not relate behaviour to elements of a structural variability model. Featured transition systems (FTS) [ [6] are an extension of labeled transition systems. Similar to feature nets, transitions are explicitly labeled with respect to a feature model, and a feature selection determines the subset of active transitions. In FTS, transitions are mapped to single features. Transition priorities are used to deal with undesired non-determinism when selecting from transitions associated to different features. With application 
conditions, priorities are no longer required because we can negate the features in other transitions to obtain the same effect.

\section{Conclusion and Future Work}

This paper introduces a modular framework for modelling systems with a high degree of variability, addressing an important challenge in software product line engineering. The modelling formalism used is feature nets, a lightweight Petri net extension in which the presence of arcs is conditional on the presence of certain features through application conditions. We present an approach to composing behavioural models from separately engineered models of individual features. Three correctness criteria for such compositions are also presented.

Feature nets capture the behaviour of entire product lines in a single, concise model, opening the way for efficient analysis and verification. We will follow this direction in future work, applying model checking techniques to our models and studying the question of verification. The practical applicability of our proposed approach will be examined in a future study, which will also determine how well the approach scales, considering that features are not always independent.

\section{References}

1. Asirelli, P., ter Beek, M.H., Gnesi, S., Fantechi, A.: A deontic logical framework for modelling product families. In: Benavides et al. [3], pp. 37-44

2. Baldan, P., Corradini, A., Ehrig, H., Heckel, R.: Compositional semantics for open Petri nets based on deterministic processes. Mathematical Structures in Computer Science 15(01), 1-35 (2005)

3. Benavides, D., Batory, D.S., Grünbacher, P. (eds.): International Workshop on Variability Modelling of Software-Intensive Systems (VaMoS), vol. 37. Universität Duisburg-Essen (2010)

4. Berthelot, G.: Transformations and decompositions of nets. In: Brauer, W., Reisig, W., Rozenberg, G. (eds.) APN 1986, Part 1. LNCS, vol. 254, pp. 359-376. Springer, Heidelberg (1987)

5. Clarke, D.: Quality Assurance for Diverse Systems, ch. 5, pp. 27-37. Deliverable 1.2 of the EternalS Coordination Action (FP7-247758), supported by the 7th Framework Programme of the EC within the FET scheme (2011), https://www.eternals.eu/sites/default/file/D1_2_TF1_stateOfTheArt.pdf

6. Classen, A., Heymans, P., Schobbens, P.Y., Legay, A., Raskin, J.F.: Model checking lots of systems: Efficient verification of temporal properties in software product lines. In: International Conference on Software Engineering, pp. 335-344. IEEE Press, Los Alamitos (2010)

7. Czarnecki, K., Antkiewicz, M.: Mapping features to models: A template approach based on superimposed variants. In: Glück, R., Lowry, M. (eds.) GPCE 2005. LNCS, vol. 3676, pp. 422-437. Springer, Heidelberg (2005)

8. Desel, J., Esparza, J.: Free choice Petri nets. Cambridge University Press, New York (1995)

9. Fantechi, A., Gnesi, S.: Formal modeling for product families engineering. In: International Software Product Line Conference, pp. 193-202. IEEE Press, Los Alamitos (2008) 
10. Farooq, U., Lam, C.P., Li, H.: Transformation methodology for UML 2.0 activity diagram into colored Petri nets. In: Advances in Computer Science and Technology, pp. 128-133. ACTA Press (2007)

11. Fischbein, D., Uchitel, S., Braberman, V.: A foundation for behavioural conformance in software product line architectures. In: International Workshop on the Role of Software Architecture in Analysis and Testing, pp. 39-48. ACM Press, New York (2006)

12. Girault, C., Valk, R.: Petri Nets for System Engineering: A Guide to Modeling, Verification, and Applications. Springer, Secaucus (2001)

13. Gruler, A., Leucker, M., Scheidemann, K.: Calculating and modeling common parts of software product lines. In: International Software Product Line Conference, pp. 203-212. IEEE Press, Los Alamitos (2008)

14. Gruler, A., Leucker, M., Scheidemann, K.: Modeling and model checking software product lines. In: Barthe, G., de Boer, F.S. (eds.) FMOODS 2008. LNCS, vol. 5051, pp. 113-131. Springer, Heidelberg (2008)

15. Larsen, K., Thomsen, B.: A modal process logic. In: Third Annual Symposium on Logic in Computer Science, pp. 203-210. IEEE Press, Los Alamitos (1988)

16. Larsen, K., Nyman, U., Wa̧sowski, A.: Modal I/O automata for interface and product line theories. In: De Nicola, R. (ed.) ESOP 2007. LNCS, vol. 4421, pp. 64-79. Springer, Heidelberg (2007)

17. Murata, T.: Petri nets: Properties, analysis and applications. Proceedings of the IEEE 77(4), 541-580 (1989)

18. Muschevici, R., Clarke, D., Proença, J.: Feature Petri Nets. In: International Software Product Line Conference, vol. 2, pp. 99-106. Lancaster University (2010)

19. Muschevici, R., Proença, J., Clarke, D.: Modular modelling of software product lines with feature nets. Tech. Rep. CW 609, KU Leuven, Belgium (2011), http://www.cs.kuleuven.be/publicaties/rapporten/cw/CW609.abs.html

20. Pohl, K., Böckle, G., van der Linden, F.: Software Product Line Engineering. Springer, Heidelberg (2005)

21. Schaefer, I.: Variability modelling for model-driven development of software product lines. In: Benavides, et al. [3], pp. 85-92

22. Schnoebelen, P., Sidorova, N.: Bisimulation and the reduction of Petri nets. In: Nielsen, M., Simpson, D. (eds.) ICATPN 2000. LNCS, vol. 1825, pp. 409-423. Springer, Heidelberg (2000)

23. Souissi, Y., Memmi, G.: Composition of nets via a communication medium. In: Rozenberg, G. (ed.) APN 1990. LNCS, vol. 483, pp. 457-470. Springer, Heidelberg (1991) 